\title{
Q.
QNEEN'S
UNIVERSITY
BELFAST
}

\section{Design of Aperiodic Frequency Selective Surfaces for Compact Quasi-Optical Networks}

Zhou, M., Sorensen, S. B., Vesterdal, N., Dickie, R., Baine, P., Montgomery, J., Cahill, R., Henry, M., Huggard, P. G., \& Toso, G. (2017). Design of Aperiodic Frequency Selective Surfaces for Compact Quasi-Optical Networks. In Proceedings of the 11th European Conference on Antennas and Propagation (EUCAP), 2017 (pp. 1274-1278). Institute of Electrical and Electronics Engineers Inc.. https://doi.org/10.23919/EuCAP.2017.7928454

\section{Published in:}

Proceedings of the 11th European Conference on Antennas and Propagation (EUCAP), 2017

\section{Document Version:}

Peer reviewed version

\section{Queen's University Belfast - Research Portal:}

Link to publication record in Queen's University Belfast Research Portal

\section{Publisher rights \\ ( $) 2017$ IEEE. Personal use of this material is permitted. Permission from IEEE must be obtained for all other uses, in any current or future media, including reprinting/republishing this material for advertising or promotional purposes, creating new collective works, for resale or redistribution to servers or lists, or reuse of any copyrighted component of this work in other works.}

\section{General rights}

Copyright for the publications made accessible via the Queen's University Belfast Research Portal is retained by the author(s) and / or other copyright owners and it is a condition of accessing these publications that users recognise and abide by the legal requirements associated with these rights.

Take down policy

The Research Portal is Queen's institutional repository that provides access to Queen's research output. Every effort has been made to ensure that content in the Research Portal does not infringe any person's rights, or applicable UK laws. If you discover content in the Research Portal that you believe breaches copyright or violates any law, please contact openaccess@qub.ac.uk. 


\title{
Design of Aperiodic Frequency Selective Surfaces for Compact Quasi-Optical Networks
}

\author{
Min Zhou ${ }^{1}$, Stig B. Sørensen ${ }^{1}$, Niels Vesterdal ${ }^{1}$, Raymond Dickie ${ }^{2}$, Paul Baine ${ }^{2}$, John Montgomery ${ }^{2}$, \\ Robert Cahill ${ }^{2}$, Manju Henry ${ }^{3}$, Peter G. Huggard ${ }^{3}$, and Giovanni Toso ${ }^{4}$ \\ ${ }^{1}$ TICRA, Copenhagen, Denmark, mz@ticra.com \\ ${ }^{2}$ Queen's University of Belfast, ECIT, Belfast, Northern Ireland, UK, r.dickie@qub.ac.uk \\ ${ }^{3}$ Rutherford Appleton Laboratory, STFC, Didcot, UK, peter.huggard@stfc.ac.uk \\ ${ }^{4}$ European Space Agency, ESTEC, Noordwijk, The Netherlands, Giovanni.Toso@esa.int
}

\begin{abstract}
The design of an aperiodic frequency selective surface (FSS) for compact quasi-optical networks is presented in this paper. Using a newly developed Physical Optics (PO) method in conjunction with a direct optimisation approach where all elements are simultaneously optimised, an FSS with an aperiodic element layout operating in several discrete bands over the frequency range $23-230 \mathrm{GHz}$ has been designed. The design procedure and a comparison with an existing periodic FSS designed to meet the same specifications are provided. This comparison indicates that enhanced performance can be obtained using an aperiodic FSS compared to a periodic FSS.
\end{abstract}

Index Terms-Frequency selective surfaces, optimisation, space applications

\section{INTRODUCTION}

Frequency selective surfaces (FSS) consist of periodic arrays of passive resonant elements, which are arranged on planar or curved surfaces [1]. The geometry of the unit cells is designed to obtain a certain spectral response and under the assumption of plane wave illumination. For space applications, FSS are widely used in various antenna systems, e.g., beamforming networks and quasi-optical networks (QON) [2].

For certain applications, it is desired to have a compact instrument, which entails the positioning of the FSS close to its source, e.g., a feed horn. In such cases, the FSS will be exposed to a large range of incidence angles and it is important that the FSS performance is maintained over a wide range of angles of incidence. One way to achieve this is the use of miniaturised FSS unit-cells [3], [4] which are less sensitive to incidence angles and can operate for non-planar phase fronts. Furthermore, reducing the size of the unit-cell is also a useful way to increase the bandwidth. An alternative novel approach exploits the larger number of degrees of freedom available from an aperiodic element layout.

The idea behind an aperiodic FSS is to optimise the elements with an approach similar to reflectarrays where each element is designed for a certain incidence angle to maintain high-performance filtering properties. Although quite intuitive, the concept of an aperiodic FSS is new and is the topic of a current ESA research/technology development contract. In this paper, we will present the design and optimisation of an aperiodic FSS to demonstrate its potential for creating compact quasi-optical feed trains for the next generation space borne remote sensing instruments.

\section{Analysis And Optimisation Methods}

\section{A. Analysis}

The RF design of traditional FSS is usually done at the unit cell level where an infinite array consisting of identical elements illuminated by a plane wave is assumed. The unit cell is then optimised to fulfil loss and bandwidths of the transmission and reflections bands from which the final design is obtained. There are several drawbacks associated with this approach. First, the finite size of the surface is not accounted for. Second, a plane wave illumination is assumed by which the near-field properties of the feed are neglected. Third, the actual dependence of the amplitude and phase of the illuminating field on incidence angle are not taken into account. These factors combined may result in an FSS design that has suboptimal performance when positioned close to the feed system. Furthermore, this methodology is not suitable for the design of aperiodic FSS. Consequently, an accurate yet computationally efficient analysis method that allows the modelling of finite sized FSS is needed. Some of the preliminary work performed by the authors in this area is presented in [5].

The analysis method used in the current work is based on a PO approach and is similar to that used in [5]. However, rather than considering all individual array elements, the FSS surface is considered as a continuous variable surface impedance, thus removing any reference to the individual array elements. For a given geometrical parameter of the array element, $a$, the distribution over the surface is considered as a function of the position and can be expressed in terms of basis spline functions

$$
a(x, y)=\sum_{i} \sum_{j} c_{i j} B_{i}(x) B_{j}(y) .
$$

Herein, the $c_{i j}$ are expansion coefficients and $B_{i}(x)$ and $B_{j}(y)$ are basis spline functions.

By applying the equivalence principle, equivalent currents are constructed on a surface enclosing the entire structure and are defined by

$$
\boldsymbol{J}_{\mathrm{S}}=\hat{n} \times \boldsymbol{H}, \quad \boldsymbol{M}_{\mathrm{S}}=-\hat{n} \times \boldsymbol{E},
$$

where $\boldsymbol{E}$ and $\boldsymbol{H}$ are the total electric and magnetic fields at the surface and $\hat{n}$ is the outward unit vector normal to that surface. Since the edge illumination of such structures are usually very 
low $(<-30 \mathrm{~dB})$, the total field at the edges are assumed to be zero and the equivalent currents are computed only on the front and rear surfaces of the structure.

For the calculation of equivalent currents, the reflection and transmission properties of the array elements are precalculated for various geometrical parameters and incidence angles, and stored in a scattering matrix look-up table. The reflection and transmission coefficients are interpolated during the analysis from which the equivalent currents at any given point on the surface can be calculated. The calculation of the reflection and transmission coefficients is done using a periodic spectral domain method of moments (SDMoM) solver [6]. Once the equivalent currents on the surface has been determined, the far-field can be calculated.

In this method, the incident field at each point on the surface must behave locally as a plane wave, otherwise the direction of incidence is not well defined and it is not possible to assign reflection and transmission coefficients to the incident field. For configurations where the FSS is located in the nearfield region of the source a plane wave expansion (PWE) of the source field is necessary in order to ensure an accurate analysis. Thus, the equivalent currents are obtained for each plane wave and subsequently added to yield the final set of currents. Although more time consuming, this is necessary if accurate results are needed.

With this PO analysis approach, all the aforementioned problems associated with the traditional FSS design approach are circumvented.

\section{B. Optimisation}

For the optimisation of the aperiodic FSS, the direct optimisation technique from [7] is used. The optimisation engine uses a gradient-based non-linear minimax optimisation algorithm which is the same algorithm used in TICRA's software POS [8].

In the direct optimisation technique in [7], all individual array elements are optimised simultaneously. This can be challenging if the FSS consists of several hundreds of thousands of elements. Optimising all individual elements simultaneously where the geometrical parameters of each element are included as optimisation variables becomes practically impossible. This issue is circumvented using the PO analysis approach where the optimisation variables are the expansion coefficients $c_{i j}$ in (1). By optimising the coefficients, one alters the geometries of the array elements over the surface.

The advantage of this approach is that the number of optimisation variables is determined by the electrical size of the FSS and not the number of array elements. However, the advantage is obtained at the cost of the restrictions imposed by the spline functions used to represent the surface since these functions cannot effectively represent discontinuities in the variation of the geometry over the surface. Such discontinuities are common in reflectarrays or transmitarrays. However, for the FSS designs, the elements are optimised to compensate for the various incidence angles, thus the geometry variation over the surface will be continuous.
As optimisation goals, different types of goals were considered for the design of the aperiodic FSS. However, for this paper we only mention one type: near-field optimisation goals. For this optimisation, the residual function represents the sum of a particular polarisation component of the electric near-field over the surface of the FSS. Thus depending on whether it is a reflective/transparent FSS, the optimisation is performed such that the transmitted/reflected near-field is minimised.

\section{FSS DESIGN SPECIFICATIONS}

To demonstrate the capabilities of an aperiodic FSS, we consider the specifications for one of the FSS designed for a prototype breadboard of the Microwave Sounder radiometer (MWS), which is one of the instruments planned for the MetOp Second Generation mission series. This FSS is denoted D1 and is the most challenging spatially demultiplexing element since it is the first FSS after the antenna, thus it must operate over the full frequency range of the instrument between $23-230 \mathrm{GHz}$ [9]. The FSS should operate at $45^{\circ}$ incidence angle and provide transmission and reflection in the bands listed in Table I.

To imitate the setup considered for the MWS prototype, we consider the configuration setup shown in Fig. 1. The FSS is circular with a diameter of $250 \mathrm{~mm}$. The FSS (centre) is positioned $130 \mathrm{~mm}$ away from the feed aperture. This position is selected to ensure a low edge illumination at least $-30 \mathrm{~dB}$ below peak.

In the transmission bands, a wideband corrugated horn designed for the MWS breadboard is used as feed. In the reflection bands, the field that illuminates the FSS does not come directly from a feed source, but rather is the reflected/transmitted field through the quasi-optical network. However, for simplicity, it is assumed that the incident field on the FSS originates from a feed source and existing feed designs operating in these bands are used.

TABLE I

TRANSMISSION AND REFLECTION FREQUENCY BANDS

\begin{tabular}{ll|lllll}
\hline $\begin{array}{c}\text { Transmission Bands } \\
{[\mathrm{GHz}]}\end{array}$ & \multicolumn{5}{c}{$\begin{array}{c}\text { Reflection Bands } \\
{[\mathrm{GHz}]}\end{array}$} \\
\hline $23.6-24.0$ & $31.3-31.5$ & $50.0-57.6$ & $87-91$ & $164-167$ & $175.3-191.3$ & $228-230$ \\
\hline
\end{tabular}

\section{APERIODIC FSS DESIGN}

The RF design of the aperiodic FSS is done in several steps. First, an FSS element is selected and optimised such that it fulfils the design specifications. Second, a scattering matrix look-up table for the element is calculated. Third, a starting point for the direct optimisation is defined. And finally, the aperiodic element layout is obtained by optimising the geometry of all the FSS array elements while taking into account the finite size of the FSS and the actual illumination across the FSS surface. 

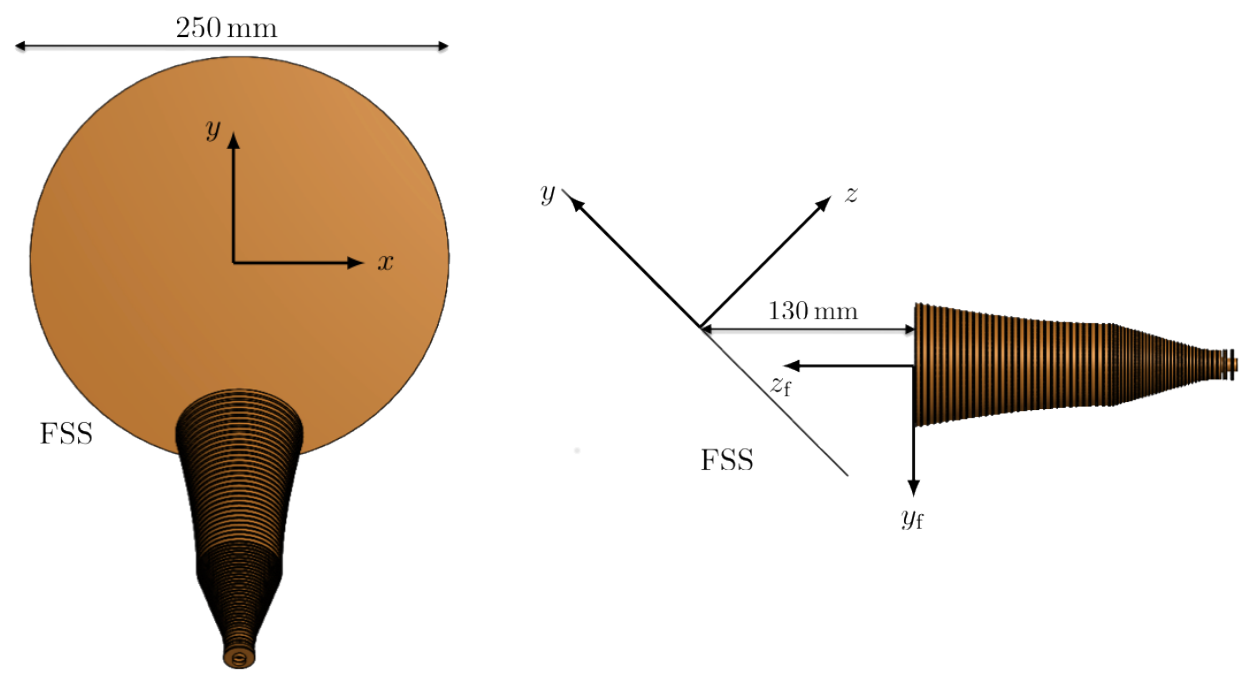

Fig. 1. The configuration considered for the design of the aperiodic FSS.

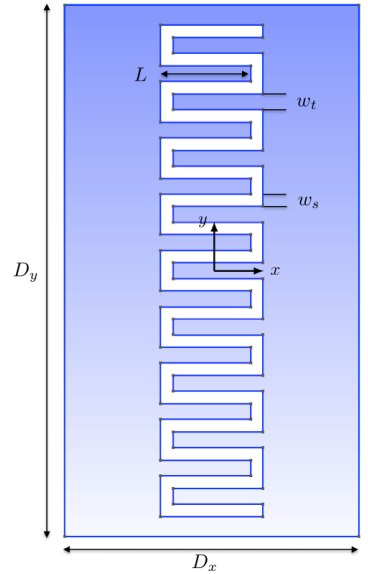

Fig. 2. Unit-cell geometry of D1. The unit-cell size is $690 \mu \mathrm{m} \times 1246 \mu \mathrm{m}$.

\section{A. FSS Element}

For the FSS element, it is apparent that the existing unit cell design from D1 [9] is an ideal candidate as it is already designed to fulfils the design specifications. The geometry of the D1 element is shown in Fig. 2. It consists of a single layer meandered slot in a metal film which is embedded between two quartz substrate layers [9]. Its spectral response is shown in Fig. 3.

\section{B. Scattering Matrix Look-up Table}

For the aperiodic FSS, the same element geometry as D1 is used. Herein, three geometrical parameters can be adjusted to fine tune the performance: slot length $L$, tooth width $w_{t}$, and slot width $w_{s}$. To arrive at an aperiodic FSS design, the three parameters are adjusted for each individual element. Consequently, the scattering matrix look-up table must contain reflection/transmission properties of the meandered slot as function of these three geometrical parameters and incidence angles for each of the frequencies of interest.

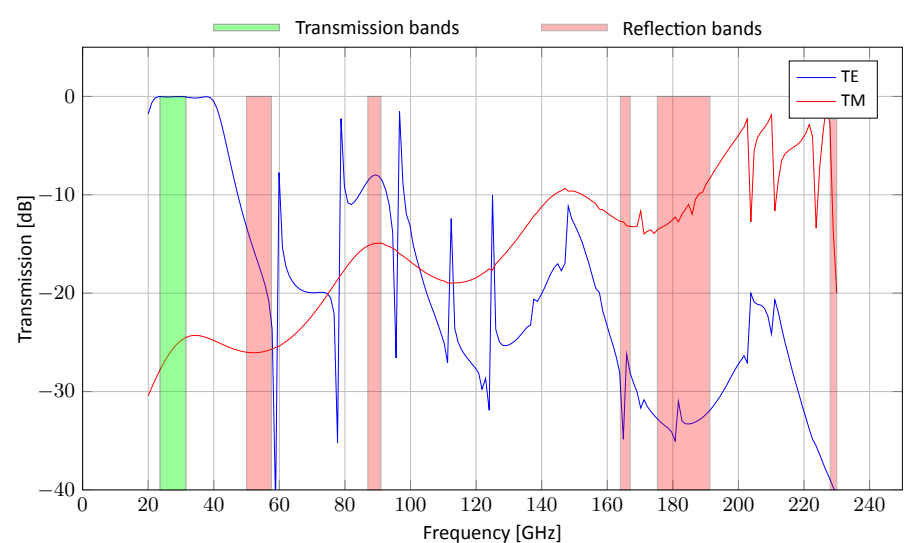

Fig. 3. Simulated transmission response of D1 at $45^{\circ}$ incidence.

Due to the complexity of the meandered slot, the computation of the look-up table is extremely time consuming and can easily take several months. Thus, it is of great importance that the number of entries needed in the scattering matrix look-up table is minimised to allow computation of the table within a reasonable time. However, the number of sampling points needs to be sufficiently high to allow accurate interpolation of the data. To reduce the number of the sampling values, the range in which the parameters are varied is decreased. For instance, the slot length $L$ can theoretically vary between $0-650 \mu \mathrm{m}$. However, only a fraction of this range may be needed for the aperiodic FSS design. Based on a number of preliminary optimisations, the ranges of the parameters has been determined and are listed in Table II.

For the aperiodic FSS, optimising the FSS in all frequency bands listed in Table I simultaneously is too demanding for the software to handle. Consequently, only the transmission bands and the first reflection band are included in the design process. These are also the frequency bands that the look-up 


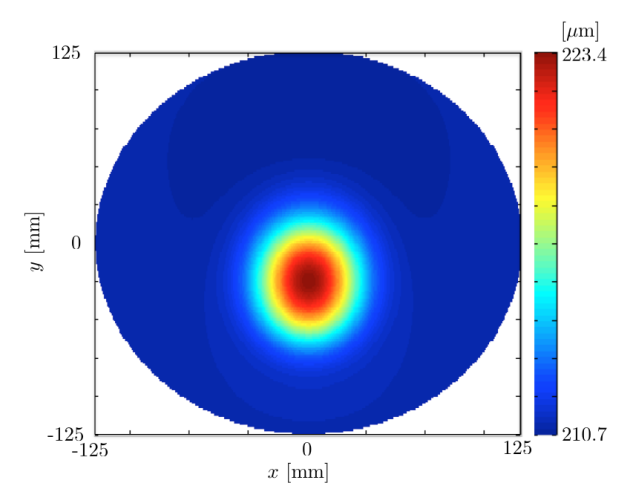

(a) $L$

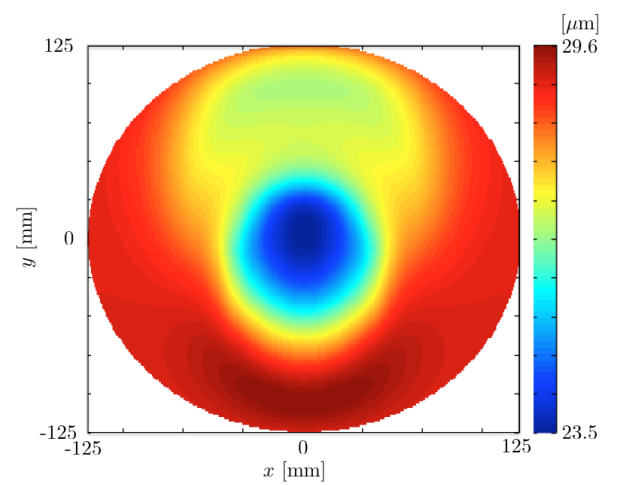

(b) $w_{s}$

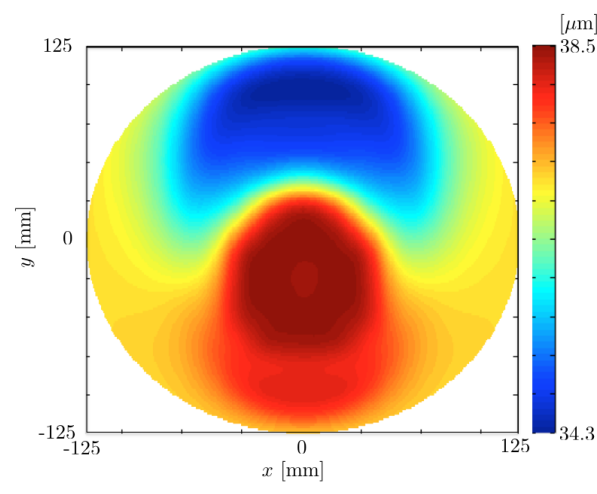

(c) $w_{t}$

Fig. 4. The layout of the elements of the aperiodic FSS. Each figure represents the variation of one of the geometrical parameters over the FSS surface, (a) $L$, (b) $w_{s}$, and (c) $w_{t}$. The colours refer to different parameter dimensions.

TABLE II

THE RANGE FOR EACH PARAMETER IN THE LOOK-UP TABLE.

\begin{tabular}{cl}
\hline Parameter & Range \\
\hline$L$ & $173-241 \mu \mathrm{m}$ \\
$w_{s}$ & $21-31 \mu \mathrm{m}$ \\
$w_{t}$ & $28-38 \mu \mathrm{m}$ \\
\hline
\end{tabular}

table computations are computed for.

\section{Initial Starting Point}

The direct optimization algorithm is gradient based, hence a good starting point is required to avoid the optimisation being trapped in a local minimum. For the aperiodic FSS, a good starting point is to use a periodic FSS that works in the intended frequency bands. The periodic D1 FSS is for instance a good candidate. However, other periodic designs could also be used. To this end, the unit-cell of the periodic D1 has been further optimised where $L, w_{t}$, and $w_{s}$ are adjusted to result in other periodic designs, which are then used as the starting point for the aperiodic FSS.

\section{Optimisation of Aperiodic FSS}

When the direct optimisation is applied on the aperiodic FSS in the transmission bands, the reflected near-field is minimised whereas in the reflection band, the transmitted near-field is minimised.

Since the geometrical parameters are represented by spline functions, the number of spline functions used to represent the geometry of the elements over the FSS determines how rapid the variations over the surface can be. By increasing the number of splines, more rapid variations over the surface can be represented. Different number of functions have been used to represent the surface. In all cases, $12 \times 12$ splines functions were found to be sufficient to represent the element variation and increasing the number did not enhance the performance.

The layout of the array elements over the optimised aperiodic FSS surface is shown in Fig. 4. Here, each figure represents one of the geometrical parameters of the straight meandered slots over the FSS surface. The different colours refer to different parameter dimensions varying from $210.7-$ $223.4 \mu \mathrm{m}$ for $L, 23.5-29.6 \mu \mathrm{m}$ for $w_{s}$, and $34.3-38.5 \mu \mathrm{m}$ for $w_{t}$.

\section{Numerical RESUlts}

In Fig. 5, the feed pattern in the presence of the periodic D1 FSS (red) and the aperiodic FSS (blue) are shown and compared to the feed pattern only (black). Looking at the curves, it is observed that most of the feed radiation is transmitted through the FSS indicating very good performance of both FSS designs, even though they are located in the nearfield region of the feed. However, around $\theta=-90^{\circ}$ some reflected fields are observed. It is seen that the reflected field from the aperiodic FSS is slightly lower compared to the periodic D1 FSS in both bands indicating that the aperiodic FSS is superior in terms of performance. Similar results are found at the other frequencies in the transmission bands.

The reflected feed pattern in the presence of the periodic D1 FSS (red), the aperiodic FSS (blue), and a metallic mirror (black) in the first two reflection bands are shown and compared in Fig. 6. It is seen that most of the feed radiation is reflected by the FSS. Similar to before, the results indicate that the aperiodic FSS is superior in terms of performance.

It is worthwhile to note that there are no additional costs associated with manufacturing an aperiodic FSS compared to periodic FSS. The aperiodic FSS is being manufactured and measurements will be performed to validate experimentally the proposed design.

\section{CONCLUSiOnS}

This paper presents the design of aperiodic frequency selective surfaces (FSS) for compact quasi-optical networks. By exploiting an aperiodic element layout, an additional number of degrees of freedom is obtained and can be used to enhance the performance of traditionally periodic FSS. Using dedicated analysis and optimisation methods, an aperiodic FSS intended for use in an atmospheric sounding instrument has been designed. The predicted performance has been compared 
$23.6 \mathrm{GHz}, \phi=90^{\circ}$

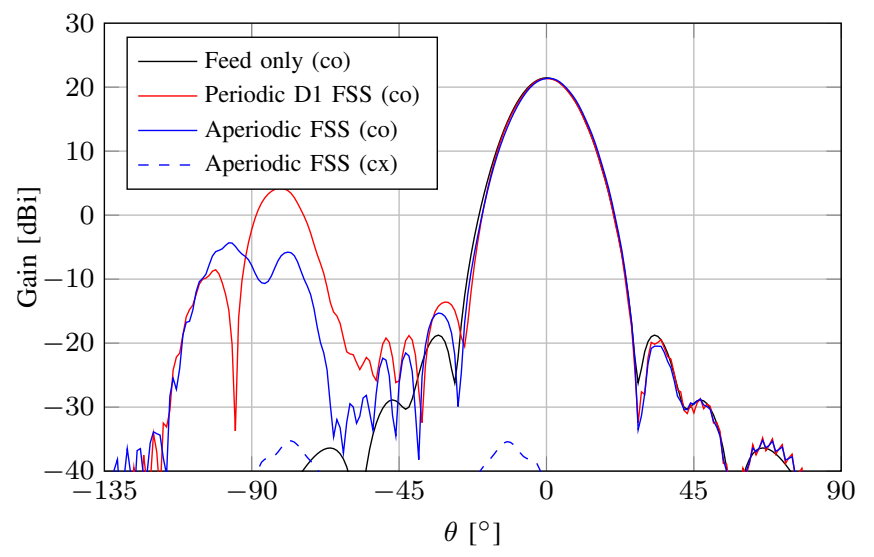

(a)
$31.3 \mathrm{GHz}, \phi=90^{\circ}$

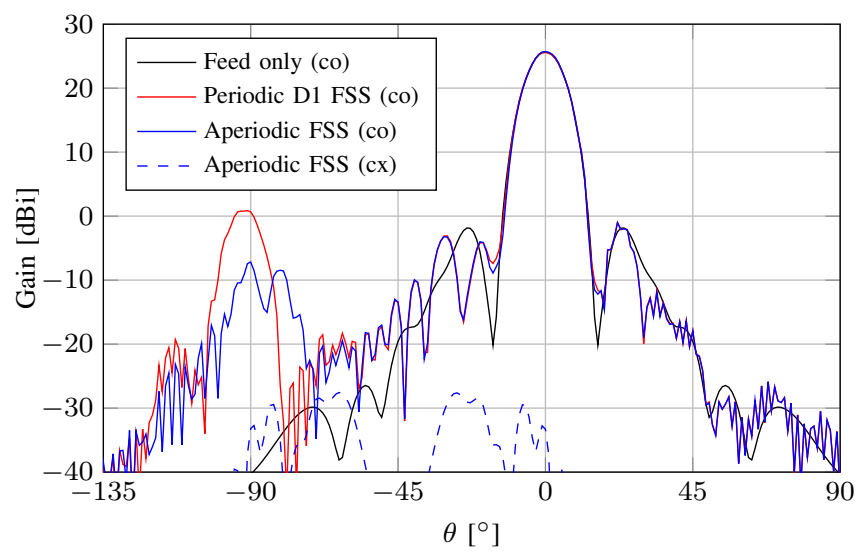

(b)

Fig. 5. The feed pattern with and without the presence of the periodic D1 FSS and the aperiodic FSS at the transmission bands. The radiation patterns are shown with respect to the feed coordinate system in Figure 1.

$53.0 \mathrm{GHz}, \phi=90^{\circ}$

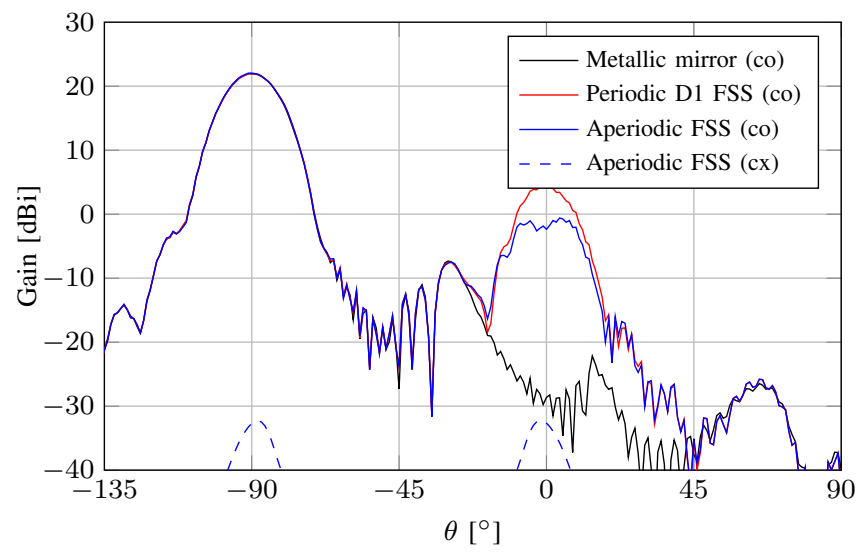

(a)

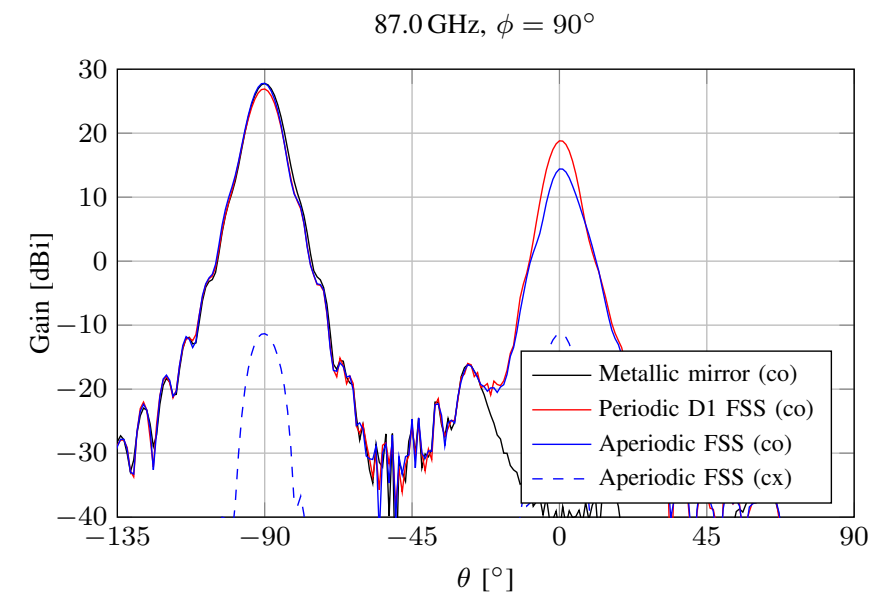

(b)

Fig. 6. The reflected feed pattern in the presence of the periodic D1 FSS, the aperiodic FSS, and a metallic mirror at the first and second reflection bands. The radiation patterns are shown with respect to the feed coordinate system in Figure 1.

to an existing periodic FSS designed for the purpose and the comparison indicates that superior performance can be achieved using an aperiodic FSS. Experimental validation is currently underway.

\section{ACKNOWLEDGMENT}

The work presented in this paper is funded by the European Space Agency (ESTEC contract No. 4000112381/14/NL/MH).

\section{REFERENCES}

[1] T. K. Wu, Frequency Selective Surface and Grid Array. Wiley Interscience, 1995.

[2] R. Dickie, R. Cahill, V. Fusco, H. S. Gamble, and N. Mitchell, "THz Frequency Selective Surface Filters for Earth Observation Remote Sensing Instruments," IEEE Trans. THz Sci. Technol., vol. 1, no. 2, pp. 450-461, 2011.

[3] K. Sarabandi and N. Behdad, "A frequency selective surface with miniaturized elements," IEEE Trans. Antennas Propag., vol. 55, no. 5, pp. $1239-1245,2007$
[4] H. Liu, K. L. Ford, and R. J. Langley, "Miniaturised bandpass frequency selective surface with lumped components," Electron. Lett., vol. 44, no. 18, pp. 1054-1055, 2008.

[5] M. Zhou, S. B. Sørensen, N. Vesterdal, R. Dickie, R. Cahill, and G. Toso, "Investigations on the analysis and design of aperiodic frequency selective surface for space applications," in Proc. 36th ESA Antenna Workshop, Noordwijk, The Netherlands, 2015.

[6] R. Mittra, C. H. Chan, and T. Cwik, "Techniques for analyzing frequency selective surfaces - a review," Proc. IEEE, vol. 76, no. 12, pp. 1593-1615, 1988.

[7] M. Zhou, S. B. Sørensen, O. S. Kim, E. Jørgensen, P. Meincke, and O. Breinbjerg, "Direct optimization of printed reflectarrays for contoured beam satellite antenna applications," IEEE Trans. Antennas Propag., vol. 61, no. 4, pp. 1995-2004, 2013.

[8] "POS Software," TICRA, Denmark, http://www.ticra.com.

[9] R. Dickie, R. Cahill, A. Mistry, M. Henry, P. G. Huggard, V. Kangas, and P. de Maagt, "Development of a 23-230 GHz frequency selective surface for the microwave sounder instrument on the MetOp second generation mission," in Proc. EuCAP, Gothenburg, Sweden, 2013. 